TITLE:

\title{
Electron diffraction of $\mathrm{ABX} 3$ perovskites with both layered ordering of A cations and tilting of BX6 octahedra
}

\section{$\operatorname{AUTHOR}(S):$}

Kishida, Kyosuke; Goto, Kengo; Inui, Haruyuki

\section{CITATION:}

Kishida, Kyosuke ...[et al]. Electron diffraction of ABX3 perovskites with both layered ordering of A cations and tilting of BX6 octahedra. Acta Crystallographica Section B: Structural Science 2009, 65(4): 405-415

\section{ISSUE DATE:}

2009-08

URL:

http://hdl.handle.net/2433/85255

RIGHT:

c 2009 International Union of Crystallography. 
Acta Crystallographica Section B

Structural

Science

ISSN 0108-7681

\section{Kyosuke Kishida, ${ }^{*}$ Kengo Goto and Haruyuki Inui}

Department of Materials Science and Engineering, Kyoto University, Sakyo-ku, Kyoto, 606-8501, Japan

Correspondence e-mail:

k.kishida@materials.mbox.media.kyoto-u.ac.jp

\section{Electron diffraction of $A B X_{3}$ perovskites with both layered ordering of $A$ cations and tilting of $B X_{6}$ octahedra}

It is shown that $21 A B X_{3}$ perovskites with tilted $B X_{6}$ octahedra and layered ordering of $A$ cations can be generated on the basis of group-subgroup relations. These structures (with 16 different space groups) are classified into ten diffraction types in terms of the conditions for superstructure reflections caused by the ordering of $A$ cations, tilting of $B X_{6}$ octahedra and structural absences. SAED (selected-area electron diffraction) allows the distinction of seven of the 21 different perovskites, while additional symmetry analysis by CBED (convergent-beam electron diffraction) is needed for the remaining 14 structures. The space groups of lithium lanthanum titanate pseudomorphs (with discrete chemical compositions) are successfully deduced by electron diffraction experiments.

\section{Introduction}

Compounds with perovskite-type structures have received attention due to their wide variety of attractive properties such as ferroelectricity, piezoelectricity, superconductivity, ionic conductivity and so on (Cava, 1990; Newnham \& Ruschau, 1991; Davies, 1999; Goodenough, 2004). The ideal crystal structure of perovskite, $A B X_{3}$ ( $A, B$ : cations; $X$ : anion), is cubic with space group $\operatorname{Pm} \overline{3} m$ (No. 221). However, the crystal structures of perovskite-type compounds are generally distorted in three different ways: tilting of $B X_{6}$ octahedra; displacement of $B$ cations; and/or distortion of the octahedra (Megaw, 1973). Additionally, if both the $A$ - and $B$-cation sites contain more than two cation types (including vacancies), an ordering of $A$ cations and/or $B$ cations may occur, also causing distortion from cubic symmetry (Woodward, 1997a,b; Mitchell, 2002; Howard et al., 2003; Howard \& Stokes, 2004, Howard \& Zhang, 2004). Since crystal structure distortion is inherently related to the physical properties of perovskites, a full examination of the factors causing structural distortions is essential.

The space-group variations caused by perovskite distortions have been studied by many researchers (Megaw, 1973; Glazer, 1972, 1975; Woodward, 1997a,b; Howard \& Stokes, 1998, 2004; Mitchell, 2002; Howard et al., 2003; Howard \& Zhang, 2004). Among the factors described above, the effect of octahedral tilting has been investigated most intensively (Glazer, 1972, 1975; Woodward, 1997a; Howard \& Stokes, 1998). The spacegroup changes associated with $B$-cation ordering such as 1:1 $\left(A_{2} B B^{\prime} X_{6}\right), 1: 2\left(A_{3} B B_{2}{ }^{\prime} X_{9}\right)$ and 1:3 $\left(A_{4} B B_{3}{ }^{\prime} X_{12}\right)$ have been examined to some extent (Woodward, 1997a; Howard \& Stokes, 2004; Howard et al., 2003; Howard \& Zhang, 2004). In contrast, $A$-cation ordering has not been systematized (Davies, 1999; Mitchell, 2002; Howard \& Zhang, 2004), as the number of perovskites showing this type of ordering is rela-
Received 29 September 2008 Accepted 30 April 2009
(C) 2009 International Union of Crystallography Printed in Singapore - all rights reserved 


\section{research papers}

Table 1

Complete list of 23 possible tilt systems used by Glazer $(1972,1975)$ and Woodward $(1997 a)$.

\begin{tabular}{|c|c|c|c|c|}
\hline $\begin{array}{l}\text { Glazer's tilt } \\
\text { system number }\end{array}$ & Glazer notation & $\begin{array}{l}\text { Relative pseudo-cubic } \\
\text { subcell parameters }\end{array}$ & $\begin{array}{l}\text { Shape of the } B X_{6} \\
\text { octahedra }\end{array}$ & Space groups \\
\hline \multicolumn{5}{|l|}{ Three-tilt systems } \\
\hline 1 & $a^{+} b^{+} c^{+}$ & $a_{\mathrm{p}} \neq b_{\mathrm{p}} \neq c_{\mathrm{p}}$ & Not distorted & $\operatorname{Immm}$ (No. 71) \\
\hline 2 & $a^{+} b^{+} b^{+}$ & $a_{\mathrm{p}} \neq b_{\mathrm{p}}=c_{\mathrm{p}}$ & Not distorted & $\operatorname{Immm}($ No. 71) \\
\hline 3 & $a^{+} a^{+} a^{+}$ & $a_{\mathrm{p}}=b_{\mathrm{p}}=c_{\mathrm{p}}$ & Not distorted & $\operatorname{Im} \overline{3}$ (No. 204) \\
\hline 4 & $a^{+} b^{+} c^{-}$ & $a_{\mathrm{p}} \neq b_{\mathrm{p}} \neq c_{\mathrm{p}}$ & Distorted & Pmmn (No. 59-2) \\
\hline 5 & $a^{+} a^{+} c^{-}$ & $a_{\mathrm{p}}=b_{\mathrm{p}} \neq c_{\mathrm{p}}$ & Distorted & $P 4_{2} / n m c$ (No. 137-2) \\
\hline 6 & $a^{+} b^{+} b^{-}$ & $a_{\mathrm{p}} \neq b_{\mathrm{p}}=c_{\mathrm{p}}$ & Distorted & Pmmn (No. 59-2) \\
\hline 7 & $a^{+} a^{+} a^{-}$ & $a_{\mathrm{p}}=b_{\mathrm{p}}=c_{\mathrm{p}}$ & Distorted & $P 4_{2} / n m c$ (No. 137-2) \\
\hline 8 & $a^{+} b^{-} c^{-}$ & $a_{\mathrm{p}} \neq b_{\mathrm{p}} \neq c_{\mathrm{p}}, \alpha \neq 90^{\circ}$ & Not distorted & $P 2_{1} / m$ (No. 11-2) \\
\hline 9 & $a^{+} a^{-} c^{-}$ & $a_{\mathrm{p}}=b_{\mathrm{p}} \neq c_{\mathrm{p}}, \alpha \neq 90^{\circ}$ & Not distorted & $P 2_{1} / m$ (No. 11-2) \\
\hline 10 & $a^{+} b^{-} b^{-}$ & $a_{\mathrm{p}} \neq b_{\mathrm{p}}=c_{\mathrm{p}}, \alpha \neq 90^{\circ}$ & Not distorted & Pnma (No. 62) \\
\hline 11 & $a^{+} a^{-} a^{-}$ & $a_{\mathrm{p}}=b_{\mathrm{p}}=c_{\mathrm{p}}, \alpha \neq 90^{\circ}$ & Not distorted & Pnma (No. 62) \\
\hline 12 & $a^{-} b^{-} c^{-}$ & $a_{\mathrm{p}} \neq b_{\mathrm{p}} \neq c_{\mathrm{p}}, \alpha \neq \beta \neq \gamma \neq 90^{\circ}$ & Not distorted & $F \overline{1}($ No. 2) \\
\hline 13 & $a^{-} b^{-} b^{-}$ & $a_{\mathrm{p}}=b_{\mathrm{p}} \neq c_{\mathrm{p}}, \alpha \neq \beta \neq \gamma \neq 90^{\circ}$ & Not distorted & I2/a (No. 15-3) \\
\hline 14 & $a^{-} a^{-} a^{-}$ & $a_{\mathrm{p}}=b_{\mathrm{p}}=c_{\mathrm{p}}, \alpha=\beta=\gamma \neq 90^{\circ}$ & Not distorted & $R \overline{3} c($ No. $167-2)$ \\
\hline \multicolumn{5}{|l|}{ Two-tilt systems } \\
\hline 15 & $a^{0} b^{+} c^{+}$ & $a_{\mathrm{p}}<b_{\mathrm{p}} \neq c_{\mathrm{p}}$ & Not distorted & Immm (No. 71) \\
\hline 16 & $a^{0} b^{+} b^{+}$ & $a_{\mathrm{p}}<b_{\mathrm{p}}=c_{\mathrm{p}}$ & Not distorted & I4/mmm (No. 139) \\
\hline 17 & $a^{0} b^{+} c^{-}$ & $a_{\mathrm{p}}<b_{\mathrm{p}} \neq c_{\mathrm{p}}$ & Not distorted & Cmcm (No. 63) \\
\hline 18 & $a^{0} b^{+} b^{-}$ & $a_{\mathrm{p}}<b_{\mathrm{p}}=c_{\mathrm{p}}$ & Not distorted & Cmcm (No. 63) \\
\hline 19 & $a^{0} b^{-} c^{-}$ & $a_{\mathrm{p}}<b_{\mathrm{p}} \neq c_{\mathrm{p}}, \alpha \neq 90^{\circ}$ & Not distorted & $I 2 / m$ (No. 12-3) \\
\hline 20 & $a^{0} b^{-} b^{-}$ & $a_{\mathrm{p}}<b_{\mathrm{p}}=c_{\mathrm{p}}, \alpha \neq 90^{\circ}$ & Not distorted & Imma (No. 74) \\
\hline \multicolumn{5}{|c|}{ One-tilt systems } \\
\hline 21 & $a^{0} a^{0} c^{+}$ & $a_{\mathrm{p}}=b_{\mathrm{p}}<c_{\mathrm{p}}$ & Not distorted & $P 4 / m b m$ (No. 127) \\
\hline 22 & $a^{0} a^{0} c^{-}$ & $a_{\mathrm{p}}=b_{\mathrm{p}}<c_{\mathrm{p}}$ & Not distorted & I4/mcm (No. 140) \\
\hline \multicolumn{5}{|l|}{ Zero-tilt system } \\
\hline 23 & $a^{\circ} a^{\circ} a^{\circ}$ & $a_{\mathrm{p}}=b_{\mathrm{p}}=c_{\mathrm{p}}$ & Not distorted & $P m s m$ (No. 221$)$ \\
\hline
\end{tabular}

perovskites containing both tilted $B X_{6}$ octahedra and layered ordering of $A$ cations.

In the present study, we examine, on the basis of group-subgroup relations, the space groups of $A B X_{3}$ perovskites when tilting of $B X_{6}$ octahedra and layered ordering of $A$ cations are simultaneously introduced. We describe how to distinguish all possible space groups for these perovskites by diffraction on the basis of systematic absences. Finally, this approach is used to analyse a lithium lanthanum titanate pseudomorphic structural pair, in which the space groups are successfully deduced by electron diffraction experiments.

\section{Glazer notation of the $B X_{6}$ octahedral tilting}

Structural variations caused by the tilting of $B X_{6}$ octahedra have been discussed in detail by Glazer (1972,

tively small. Recently, Howard \& Zhang (2004) briefly reported the possible space-group variations of perovskites with layered ordering of $A$ cations through group theoretical analysis. However, less detail was given about the crystallographic parameters, including the Wyckoff positions of the constituent ions, which can be used in diffraction experiments.

Recently, $\mathrm{Ln}_{2 / 3} \mathrm{TiO}_{3}, \mathrm{Ln}_{1 / 3} \mathrm{NbO}_{3}$ and $\mathrm{Ln}_{1 / 3} \mathrm{TaO}_{3} \quad(\mathrm{Ln}=$ lanthanoid) perovskites that contain $A$-cation ordering have been of interest as hosts for Li-ion insertion and extraction in electrodes or solid electrolytes in lithium secondary batteries (Inaguma et al., 1993, 2002; Carrillo et al., 2000; Nakayama et al., 2003; Howard \& Zhang, 2004; Kishida et al., 2007a,b; Catti et al., 2007). Among these perovskites, Li-substituted $\mathrm{La}_{2 / 3} \mathrm{TiO}_{3}\left[\mathrm{La}_{2 / 3-x} \mathrm{Li}_{3 x} \mathrm{TiO}_{3}\right.$ (LLT) $]$ is the most promising electrolyte in all-solid-state lithium batteries because of its very high ionic conductivity of $1.1 \times 10^{-3} \mathrm{~S} \mathrm{~cm}^{-1}$ at room temperature $(x \simeq 0.1)$ and temperature stability (Inaguma $e t$ al., 1993). Since the ionic conductivity of LLT strongly depends on its chemical composition, and thereby its crystal structure, extensive structure determinations have been conducted (Stramare et al., 2003). These reveal that $\mathrm{La}^{3+}$ ordering is layered, such that $\mathrm{La}^{3+}$-rich and $\mathrm{La}^{3+}$-poor $A$ cation layers stack alternately along one of the pseudo-cubic axes. In addition, tilting of $\mathrm{TiO}_{6}$ octahedra occurs (Inaguma et al., 2002; Stramare et al., 2003; Kishida et al., 2007a; Catti et al., 2007). However, the crystal structures of the LLT phases remain controversial. We thus believe it is timely to establish a systematic method for determining the space groups of
1975), Megaw (1973), Woodward (1997a) and Howard \& Stokes (1998). Glazer $(1972,1975)$ proposed a simple method and notation to describe possible tilt systems in perovskites with components around the cubic axes of the ideal structure. Glazer notation has become a standard method for describing $B X_{6}$ tilt systems, using three superscripts, $a^{\#} b^{\#} c^{\#}$. The three characters represent a tilt axis (either the [100], [010] or [001] axis) of a cubic perovskite cell, with the superscript \# taking either $0,+$ or - , to indicate no tilt, the tilt of successive octahedra in the same sense (in-phase tilting), or in the opposite sense (anti-phase tilting). Repetition of the same character, such as $a^{\#} a^{\#} c^{\#}$, indicates that the tilt magnitudes are identical. Glazer deduced 23 different tilt systems (Table 1; Glazer, 1972, 1975; Woodward, 1997a), some of which are assigned the same space group. For example, the tilt systems $a^{0} b^{+} b^{-}$and $a^{0} b^{+} c^{-}$conform to $C m c m$. The number of different space groups is thus reduced to 16. Glazer $(1972,1975)$ deduced the 23 tilt systems assuming that $B X_{6}$ octahedra are perfectly rigid (not distorted), but Leinenweber \& Parise (1995), Woodward (1997a) and Howard \& Stokes (1998) reconsidered the assignment of symmetry, taking into account the necessity of $B X_{6}$ corner linking and found that in four tilt systems, $a^{+} b^{+} c^{-}, a^{+} a^{+} c^{-}, a^{+} b^{+} b^{-}$and $a^{+} a^{+} a^{-}$, the octahedra are necessarily distorted, with the result that the space groups are different from those of Glazer (1972, 1975). The atomic positions and unit-cell descriptions for all 16 space groups generated by the tilting of $B X_{6}$ octahedra are summarized by Woodward (Table 5 in Woodward, 1997a). 
Table 2

List of possible derivative tilt systems with layered ordering of $A$ cations.

The axis along which layered ordering occurs is expressed with a capital letter in the Glazer notation.

\begin{tabular}{|c|c|c|c|c|c|}
\hline \multirow[b]{2}{*}{$\begin{array}{l}\text { Glazer's tilt } \\
\text { system number }\end{array}$} & \multirow[b]{2}{*}{ Space group } & \multirow[b]{2}{*}{$\begin{array}{l}\text { Representative tilt } \\
\text { system after Glazer }\end{array}$} & \multicolumn{3}{|c|}{ Tilt systems with layered ordering of $A$ cations } \\
\hline & & & Along $a$ axis & Along $b$ axis & Along $c$ axis \\
\hline $1,2,15$ & Immm (No. 71) & $a^{+} b^{+} c^{+}$ & $A^{+} b^{+} c^{+}(1-1)$ & $\leftarrow$ & $\leftarrow$ \\
\hline 3 & $\operatorname{Im} \overline{3}$ (No. 204) & $a^{+} a^{+} a^{+}$ & $\uparrow$ & $\leftarrow$ & $\leftarrow$ \\
\hline 4,6 & Pmmn (No. 59:2) & $a^{+} b^{+} c^{-}$ & $A^{+} b^{+} c^{-}(4-1)$ & $\leftarrow$ & $a^{+} b^{+} C^{-}(4-2)$ \\
\hline 5,7 & $P 4_{2} / n m c$ (No. 137:2) & $a^{+} a^{+} c^{-}$ & $\uparrow$ & $\leftarrow$ & $a^{+} a^{+} C^{-}(5-1)$ \\
\hline 8,9 & $P 2_{1} / m($ No. $11: b 1)$ & $a^{+} b^{-} c^{-}$ & $A^{+} b^{-} c^{-}(8-1)$ & $a^{+} B^{-} c^{-}(8-2)$ & $\leftarrow$ \\
\hline 10,11 & Pnma (No. 62) & $a^{+} b^{-} b^{-}$ & $A^{+} b^{-} b^{-}(10-1)$ & $\uparrow$ & $\leftarrow$ \\
\hline 12 & $F \overline{1}($ No. 2$)$ & $a^{-} b^{-} c^{-}$ & $A^{-} b^{-} c^{-}(12-1)$ & $\leftarrow$ & $\leftarrow$ \\
\hline 13 & I2/a (No. 15:b3) & $a^{-} b^{-} b^{-}$ & $A^{-} b^{-} b^{-}(13-1)$ & $\uparrow$ & $\leftarrow$ \\
\hline 14 & $R \overline{3} c($ No. $167: 2)$ & $a^{-} a^{-} a^{-}$ & $\uparrow$ & $\leftarrow$ & $\leftarrow$ \\
\hline 16 & I4/mmm (No. 139) & $a^{0} b^{+} b^{+}$ & $A^{0} b^{+} b^{+}(16-1)$ & $a^{0} B^{+} c^{+}(16-2)$ & $\leftarrow$ \\
\hline 17,18 & Cmcm (No. 63) & $a^{0} b^{+} c^{-}$ & $A^{0} b^{+} c^{-}(17-1)$ & $a^{0} B^{+} c^{-}(17-2)$ & $a^{0} b^{+} C^{-}(17-3)$ \\
\hline 19 & $I 2 / m$ (No. 12:b3) & $a^{0} b^{-} c^{-}$ & $A^{0} b^{-} c^{-}(19-1)$ & $a^{0} B^{-} c^{-}(19-2)$ & $\leftarrow$ \\
\hline 20 & Imma (No. 74) & $a^{0} b^{-} b^{-}$ & $A^{0} b^{-} b^{-}(20-1)$ & $\uparrow$ & $\leftarrow$ \\
\hline 21 & $P 4 / m b m$ (No. 127) & $a^{0} a^{0} c^{+}$ & $A^{0} b^{0} c^{+}(21-1)$ & $\leftarrow$ & $a^{0} a^{0} C^{+}(21-2)$ \\
\hline 22 & $I 4 / m c m$ (No. 140) & $a^{0} a^{0} c^{-}$ & $A^{0} b^{0} c^{-}(22-1)$ & $\leftarrow$ & $a^{0} a^{0} C^{-}(22-2)$ \\
\hline 23 & $P m \overline{3} m$ (No. 221) & $a^{0} a^{0} a^{0}$ & $\rightarrow$ & $\rightarrow$ & $a^{0} a^{0} C^{0}(23-1)$ \\
\hline
\end{tabular}

Woodward aristotypes and summarized with the extended Glazer notation is determined to be 22 (Table 2).

We now consider the space groups for these 22 different hettotypes on the basis of groupsubgroup relationships. The occurrence of layered ordering of $A$ cations must result in split Wyckoff positions for the $A$ cations along one of the pseudocubic axes. Therefore, the maximal subgroups are chosen that allow site splitting for the aristotype space group. The subgroup with the highest symmetry from these maximal subgroups is selected as the space group of the hettotype. If none of the maximal subgroups of the aristotype allow the required site splitting, subgroups of the

\section{Space-group variations of perovskites by introducing layered ordering of $A$ cations in addition to $B X_{6}$ octahedral tilting}

As initial basic crystal structures (aristotypes), we take the 16 tilt systems with unique space groups (Woodward, 1997a), and consider geometrically possible variations of the space groups by introducing layered ordering of $A$ cations on the basis of group-subgroup relations. $A$-cation ordering is assumed to occur along a pseudo-cubic axis of the ideal perovskite structure. For ease of understanding, we express the axis along which the layered ordering occurs with a capital letter in Glazer's notation. For example, when the tilt system $a^{0} a^{0} c^{+}$ possesses $A$-cation ordering along the $c$ axis, the system is expressed as $a^{0} a^{0} C^{+}$; we call this extended Glazer notation. The number of derivative systems (hettotypes) basically depends on the number of distinguishable pseudo-cubic axes of their aristotype with respect to the tilt types. When the aristotype possesses pseudo-cubic axes with three different tilt types, i.e. zero-, in-phase and anti-phase tilts (for example, $a^{0} b^{+} c^{-}$), three hettotypes must be considered with distinguishable pseudo-cubic axes. For an aristotype with two different tilts, it is sufficient to consider two hettotypes. For $a^{+} b^{-} c^{-}$, for example, three different types of hettotypes expressed with the extended Glazer notation of $A^{+} b^{-} c^{-}$, $a^{+} B^{-} c^{-}$and $a^{+} b^{-} C^{-}$can be generated. However, the latter two hettotypes turn out to be identical if the setting of the coordinate system is changed, i.e. the $b$ and $c$ axes are interchangeable and the tilt systems of $A^{+} b^{-} c^{-}$and $a^{+} B^{-} c^{-}$are sufficient. When the tilt types for all pseudo-cubic axes are identical, only one hettotype is necessary, except for the aristotype $a^{-} b^{-} b^{-}$, where $A^{-} b^{-} b^{-}$and $a^{-} B^{-} c^{-}$(equivalent to $A^{-} b^{-} c^{-}$) can be considered. The number of all possible hettotypes thus generated from the 16 different Glazer- maximal subgroups are inspected and the procedure repeated until an appropriate subgroup is found.

Here we examine, for example, the space groups of hettotypes generated by introducing the layered ordering of $A$ cations along one of the pseudo-cubic axes of the aristotype $a^{0} a^{0} c^{-}$[space group: $I 4 / m c m$ (No. 140)]. This tilt system possesses tetragonal symmetry with the $2^{1 / 2} a_{\mathrm{p}} \times 2^{1 / 2} a_{\mathrm{p}} \times 2 a_{\mathrm{p}}$ diagonal-type unit cell with the lattice vectors corresponding to [110], [110] and [002] of the ideal perovskite structure. In $I 4 / \mathrm{mcm}$, the $A$ cations occupy $4 b$ sites, whose atomic coordinates are $(0,1 / 2,1 / 4)$. Of the maximal subgroups of $14 / \mathrm{mcm}$, only P4/nbm (No. 125: origin choice 2) allows the required splitting of $4 b$ Wyckoff positions, and therefore is chosen as the hettotype space group (Aroyo et al., 2006). The extended Glazer notation is $a^{0} a^{0} C^{-}$. The correspondence of Wyckoff positions and their site symmetry for $14 / \mathrm{mcm}$ to those for $P 4 / \mathrm{nbm}$ can be written as follows, accompanied by the shift of the origin by $1 / 4,1 / 4,1 / 4$ :

$$
\begin{gathered}
A \text { site }: 4 b(-42 m) \rightarrow 2 c(-42 m), 2 d(-42 m), \\
B \text { site }: 4 c(4 / m . .) \rightarrow 4 g(4 . .), \\
X \text { site (i) }: 4 a(422) \rightarrow 2 a(422), 2 b(422), \\
X \text { site (ii) }: 8 h(m .2 m) \rightarrow 8 m(. . m) .
\end{gathered}
$$

For the $a^{0} a^{0} c^{-}$aristotype, another possible configuration of $A$-cation ordering is along one of the cubic axes perpendicular to the $c$ axis. In this case, the tetragonal symmetry of the aristotype is lost because the $a$ axis and $b$ axis are no longer equivalent, and thus the hettotype is expressed as $A^{0} b^{0} c^{-}$ (Table 2). However, none of the maximal subgroups of the space group of $14 / \mathrm{mcm}$ allow the required site splitting and, as a result, the space group of Cmmm (No. 65) is chosen, since it exhibits the highest symmetry among the subgroups that meet 


\section{research papers}

Table 3

Space groups, approximate unit-cell dimensions and reflection conditions for the 22 possible hettotypes listed in Table 2 .

The extended Glazer notations for some hettotypes are modified to be consistent with the standard settings in International Tables for Crystallography (Hahn, 2002).

\begin{tabular}{|c|c|c|c|c|c|}
\hline \multirow[b]{2}{*}{$\begin{array}{l}\text { Tilt system } \\
\text { number }\end{array}$} & \multirow[b]{2}{*}{$\begin{array}{l}\text { Extended Glazer } \\
\text { notation }\end{array}$} & \multirow[b]{2}{*}{ Space group } & \multirow{2}{*}{$\begin{array}{l}\text { Approximate } \\
\text { unit-cell } \\
\text { dimensions }\end{array}$} & \multicolumn{2}{|l|}{ Reflection conditions } \\
\hline & & & & General conditions & Additional conditions \\
\hline $1-1$ & $A^{+} b^{+} c^{+}$ & Pmmm (No. 47) & $2 \times 2 \times 2$ & No conditions & $h k l: h+k+l=2 n$ or $h k l: k+l=2 n$ \\
\hline $4-1$ & $A^{+} b^{+} c^{-}$ & Pmm2 (No. 25) & $2 \times 2 \times 2$ & No conditions & - \\
\hline $4-2$ & $a^{+} b^{+} C^{-}$ & Pmm2 (No. 25) & $2 \times 2 \times 2$ & No conditions & - \\
\hline $5-1$ & $a^{+} a^{+} C^{-}$ & $P \overline{4} m 2$ (No. 115$)$ & $2 \times 2 \times 2$ & No conditions & - \\
\hline $16-1$ & $a^{+} a^{+} C^{0}$ & P4/mmm (No. 123) & $2 \times 2 \times 2$ & No conditions & $h k l: h+k+l=2 n$ or $h k l: h+k=2 n$ \\
\hline $16-2$ & $a^{0} B^{+} c^{+}$ & Pmmm (No. 47) & $2 \times 2 \times 2$ & No conditions & $h k l: h+k+l=2 n$ or $h k l: h+l=2 n$ \\
\hline $8-1$ & $a^{-} B^{+} c^{-}$ & $\operatorname{Pm}($ No. $6: b)$ & $2^{1 / 2} \times 2 \times 2^{1 / 2}$ & No conditions & - \\
\hline $19-1$ & $a^{-} B^{0} c^{-}$ & $P 2 / m$ (No. $10: b)$ & $2^{1 / 2} \times 2 \times 2^{1 / 2}$ & No conditions & - \\
\hline $12-1$ & $a^{-} b^{-} C^{-}$ & $C \overline{1}($ No. 2) & $2 \times 2 \times 2$ & $h k l: h+k=2 n$ & - \\
\hline $17-2$ & $A^{+} b^{0} c^{-}$ & $A m m 2$ (No. 38$)$ & $2 \times 2 \times 2$ & $h k l: k+l=2 n$ & - \\
\hline $19-2$ & $A^{-} b^{0} c^{-}$ & $A 2 / m$ (No. 12:b2) & $2 \times 2 \times 2$ & $h k l: k+l=2 n$ & - \\
\hline $22-1$ & $a^{0} b^{-} C^{0}$ & Cmmm (No. 65) & $2 \times 2 \times 2$ & $h k l: h+k=2 n$ & - \\
\hline $10-1$ & $A^{+} b^{-} b^{-}$ & $P m c 2_{1}$ (No. 26) & $2 \times 2^{1 / 2} \times 2^{1 / 2}$ & $h 0 l: l=2 n 00 l: l=2 n$ & - \\
\hline $13-1$ & $A^{-} b^{-} b^{-}$ & $P 2 / c$ (No. 13:b1) & $2 \times 2^{1 / 2} \times 2^{1 / 2}$ & $h 0 l: l=2 n 00 l: l=2 n$ & - \\
\hline $20-1$ & $a^{-} B^{0} a^{-}$ & Pmma (No. 51) & $2^{1 / 2} \times 2 \times 2^{1 / 2}$ & $h k 0: h=2 n h 00: h=2 n$ & - \\
\hline $8-2$ & $A^{-} b^{+} c^{-}$ & $P 2_{1} / m$ (No. $\left.11: b\right)$ & $2 \times 2 \times 2$ & $0 k 0: k=2 n$ & - \\
\hline $17-1$ & $a^{+} B^{0} c^{-}$ & Pmma (No. 51) & $2 \times 2 \times 2$ & $h k 0: h=2 n h 00: h=2 n$ & - \\
\hline $17-3$ & $a^{+} b^{0} C^{-}$ & Pmma (No. 51) & $2 \times 2 \times 2$ & $h k 0: h=2 n h 00: h=2 n$ & - \\
\hline $21-1$ & $A^{0} b^{0} c^{+}$ & Pmmm (No. 47) & $2 \times 2 \times 1$ & No conditions & $h k l: k=2 n$ or $h k l: h+k=2 n(h \neq \pm k)$ \\
\hline $21-2$ & $a^{0} a^{0} C^{+}$ & P4/mbm (No. 127) & $2^{1 / 2} \times 2^{1 / 2} \times 2$ & $0 k l: k=2 n h 00: h=2 n h k l: h+k=2 n$ & - \\
\hline $22-2$ & $a^{0} a^{0} C^{-}$ & P4/nbm (No. 125:2) & $2^{1 / 2} \times 2^{1 / 2} \times 2$ & $h k 0: h+k=2 n 0 k l: k=2 n h 00: h=2 n$ & $h k l: h+k+l=2 n$ or $h k l: h+k=2 n$ \\
\hline 23-1 & $a^{0} a^{0} C^{0}$ & P4/mmm (No. 123) & $1 \times 1 \times 2$ & No conditions & - \\
\hline
\end{tabular}

this requirement. The crystallographic parameters (atomic positions and unit-cell descriptions) of the $P 4 / \mathrm{nbm}$ and $\mathrm{Cmmm}$ hettotypes, generated from $I 4 / \mathrm{mcm}$, are summarized in the supplementary material ${ }^{\mathbf{1}}$.

The analysis of all 22 hettotypes with the space groups described in the standard settings from International Tables for Crystallography (Hahn, 2002), following Woodward (1997a), are summarized in Table 3. Accordingly, the extended Glazer notations in Table 2 were modified to be consistent with the standard settings used in Table 3. The unit-cell dimensions and the corresponding reflection conditions of these space groups are collected in Table 3. Among the 22 hettotypes, $A^{+} b^{+} c^{+}$and $a^{0} B^{+} c^{+}$with the $2 a_{\mathrm{p}} \times 2 a_{\mathrm{p}} \times 2 a_{\mathrm{p}}$ type unit cell belong to the same space group, Pmmm (No. 47), the difference being whether the tilt angle along one of the pseudo-cubic axes is set to zero. From the viewpoint of Pmmm symmetry, the tilt is not necessarily zero, so it is sufficient to only consider the tilt system $A^{+} b^{+} c^{+}$. Thus, the number of the different hettotypes is reduced to 21 . Crystallographic parameters of all these 21 hettotypes are summarized in the supplementary material, where 16 space groups are assigned to the 21 hettotypes on the basis of group-subgroup relations. The result of the space-group analysis for hettotypes (Table 3) is essentially consistent with group-theoretical analysis (Howard \& Stokes, 1998), except for $A^{+} b^{+} c^{-}$and $a^{+} b^{+} C^{-}$ which are missing in their analysis. This is simply because Howard \& Stokes (1998) have excluded the space group

\footnotetext{
${ }^{1}$ Supplementary data for this paper are available from the IUCr electronic archives (Reference: WH5002). Services for accessing these data are described at the back of the journal.
}

Pmmn for two aristotypes $a^{+} b^{+} c^{-}$and $a^{+} b^{+} b^{-}$, because their analysis suggested that the tilt of $c^{-}$and $b^{-}$in these aristotypes would not be simple anti-phase tilting.

\section{Space-group determination by electron diffraction}

\subsection{Structural absences occurring for particular hettotypes}

Extinction conditions for the 21 hettotypes are readily obtained from the International Tables for Crystallography (Hahn, 2002) based on the space groups assigned in the previous section. For space-group determination, it is sufficient in many cases to consider only general and special conditions, the former of which are always obeyed irrespective of which Wyckoff positions are occupied, while the latter apply only to special Wyckoff positions and occur in addition to the general conditions. However, so-called 'structural (or non-space-group) absences' may also occur if atoms in a particular crystal structure make a special arrangement so that 'false symmetry' appears (Hahn, 2002). We have found that structural absences occur in the reflection conditions for some of the 21 different hettotypes, as exemplified below.

We first consider hettotype $a^{0} a^{0} C^{-}$(space group $P 4 / n b m$ : No. 125 , origin choice 2) where the general reflection conditions for the space group are: $h k 0: h+k=2 n, 0 k l: k=2 n, h 00$ : $h=2 n$. Of the occupied Wyckoff positions $2 a, 2 b, 2 c, 2 d, 4 g$ and $8 m$ (supplementary material), the special conditions $h k l: h$ $+k=2 n$ apply to the first five, while there is no special condition for $8 \mathrm{~m}$. Therefore, no additional conditions should occur by considering the union of the special conditions because the $8 m$ site is occupied by $X$ anions. In other words, if 


\section{Table 4}

List of superstructure reflections observed in SAED patterns taken along some low-indexed directions of the 21 different hettotypes.

The subscript $\mathrm{p}$ is used to refer to the pseudo-cubic unit cell corresponding to the ideal perovskite structure. Indices $o$ and $e$ indicate odd and even numbers. The extended Glazer notations for some hettotypes are modified from those listed in Tables 2 and 3 in such a way that the layered ordering of $A$ cations always occurs along the $c$ axis of the ideal perovskite structure for any hettotypes.

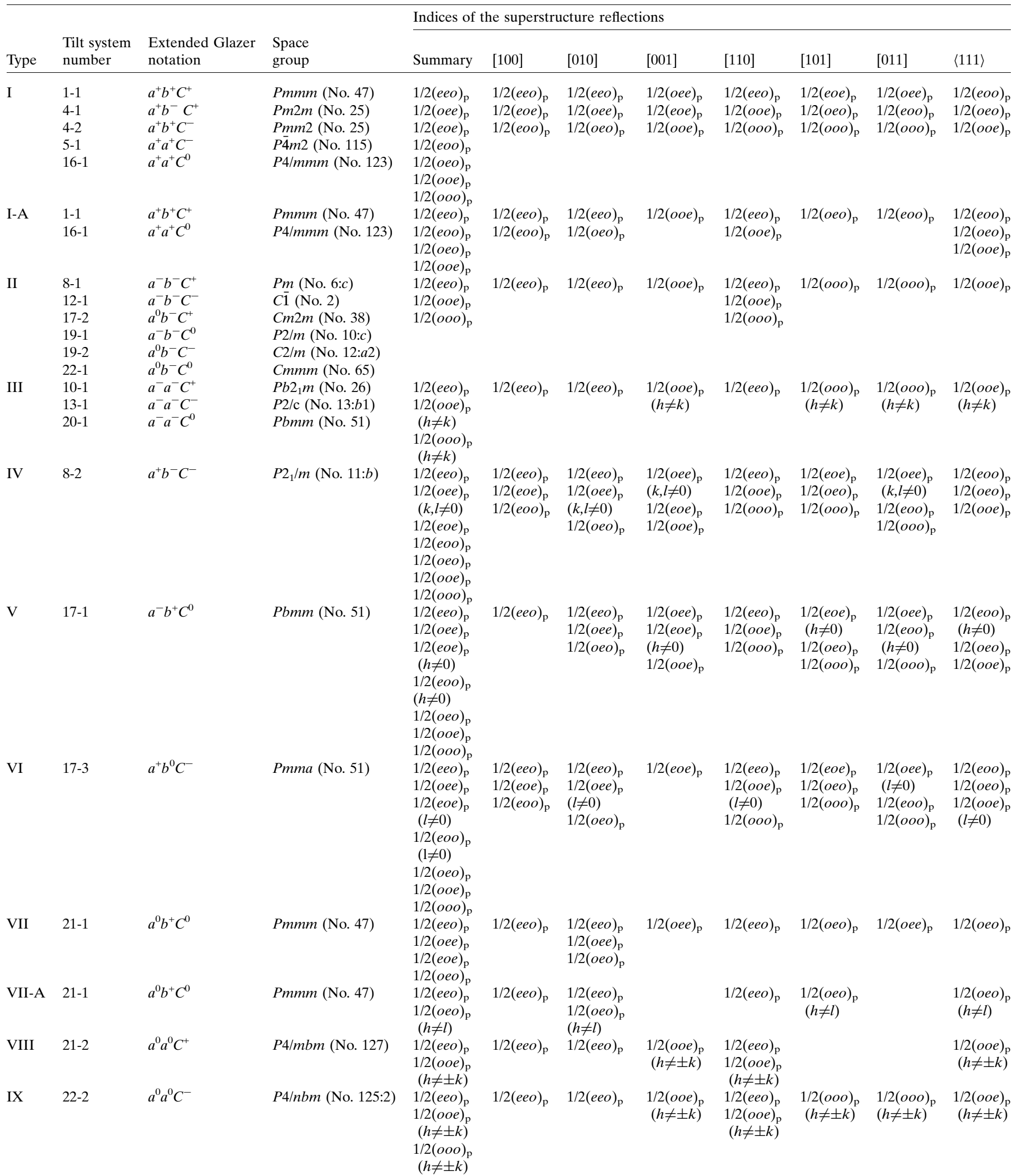




\section{research papers}

Table 4 (continued)

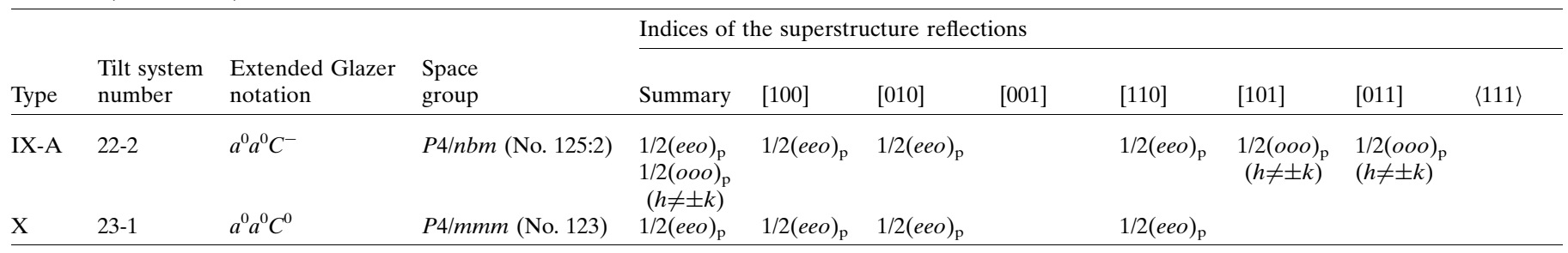

the $X$ anions in the $8 m$ site were in a special arrangement so that 'false symmetry' was exhibited, an additional reflection condition would appear. In the present case, the $B X_{6}$ octahedron is assumed to be rigid (undistorted), i.e. the $X$ anions in $8 m$ sites are assumed to take a special value (1/4) of the $z$ coordinate [ $8 m$ sites: $(x+1 / 2, x, z)]$. Under this assumption, body-centring results, giving rise to 'structural absences', i.e. $h k l: h+k+l=2 n$, is an extra condition for the $X$ anions. Similarly, the simultaneous occupancy of both the $2 a$ and $2 b$ sites by $X$ anions generates body-centring for the relevant ions. For this case, the crystal structure for the $a^{0} a^{0} C^{-}$ perovskite with rigid (undistorted) $B X_{6}$ octahedra will possess an additional reflection condition corresponding to a union of the special condition $h k l: h+k=2 n$ and the extra condition $h k l: h+k+l=2 n$; this extinction condition is the case for Lirich LLT as described in $\$ 5.1$.

We now consider which hettotypes have high probabilities for the occurrence of additional reflection conditions caused by structural absences. If $A$ cations are assumed to be located at the corners of the pseudo-cubic unit cell, they will generate one-face-centring on the plane where the layered ordering of $A$ cations occurs. Then, the hettotypes of $A^{+} b^{+} c^{+}$and $a^{+} a^{+} C^{0}$
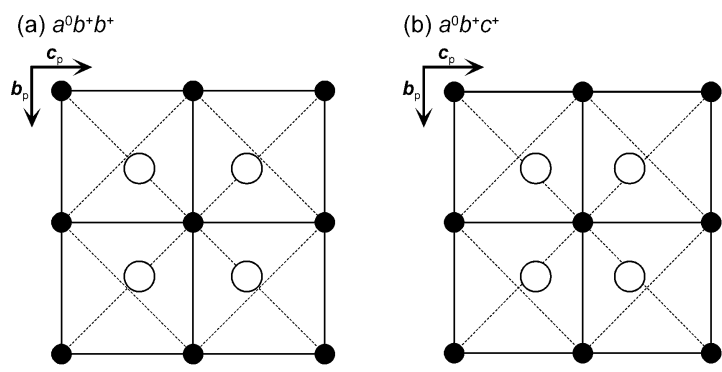

(c) $a^{0} b^{+} c^{-}$

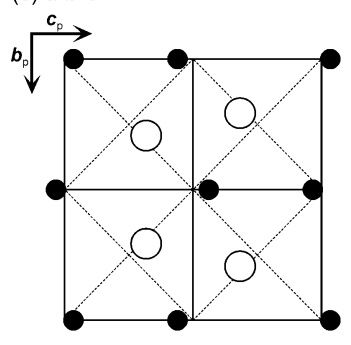

(d) $a^{0} b-c$

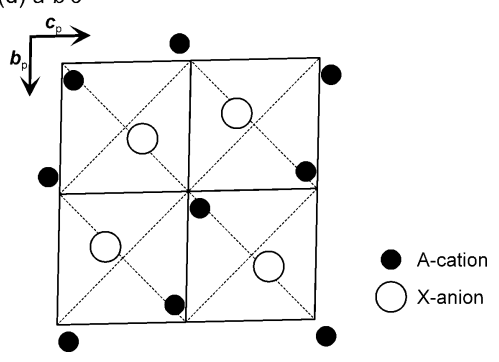

Figure 1

Atomic arrangement of (100) for four different types of tilt systems, namely $(a) a^{0} b^{+} b^{+},(b) a^{0} b^{+} c^{+},(c) a^{0} b^{+} c^{-}$and $(d) a^{0} b^{-} c^{-}$. Filled and open circles correspond to $A$ cations and $X$ anions, respectively. The unit cell for each tilt system is shown by thick lines. Atomic displacements are only approximate and are exaggerated to exhibit their effects more clearly. have high probabilities for extra systematic absences, since they possess more than two in-phase tilt axes without an antiphase tilt axis. This can easily be understood by considering the shift of $X$ anions located at the apexes of $B X_{6}$ octahedra, all of which are located on the same pseudo-cubic plane (Fig. 1). As a result of the $B X_{6}$ tilting, these shifts occur as the Glazer notation indicates. For the two hettotypes described above, these shifts occur symmetrically with respect to the corner of the pseudo-cubic unit cell (Figs. $1 a$ and $b$ ). It is then quite reasonable for $A$ cations to be located exactly at the corner of the pseudo-cubic unit cell, giving rise to the oneface-centring. For hettotypes possessing at least one antiphase tilt axis, on the other hand, these shifts occur asymmetrically with respect to the corner of the pseudo-cubic unit cell (Figs. $1 c$ and $d$ ) and may lead to the shift of $A$ cations and destruction of the centre. Therefore, we do not consider the possibility of the occurrence of additional reflection conditions for other hettotypes (Table 3).

\subsection{Electron diffraction method for space-group determina- tion of 21 hettotypes}

Although unit-cell dimensions vary from hettotype to hettotype (Table 3), it is not practical to use these in electron diffraction analysis since the indices for reflections also vary with the unit-cell dimension. We therefore employ the pseudocubic unit cell corresponding to the ideal perovskite structure as the standard unit cell used for electron diffraction analysis, as proposed by Glazer $(1972,1975)$ and Woodward $(1997 a)$. With this standard unit-cell setting, reflections corresponding to the ideal cubic perovskite can be regarded as fundamental reflections, whereas reflections caused by octahedral tilting and $A$ layering are superstructure reflections. The reflection conditions for superstructure reflections in Table 4 are converted from those in Table 3 so as to fit the standard unitcell setting. Superstructure reflections are expressed in general as non-integers, although the fundamental reflections of the cubic perovskite are always integers. In Table 4 the unit-cell setting for some hettotypes is changed from the standard setting used in Table 3 in such a way that the layered $A$ cations always occur along the $c$ axis of the ideal perovskite. With this change of unit-cell setting, superstructure indices are unambiguously designated to facilitate electron diffraction analysis. If we ignore slight orthorhombic or monoclinic lattice distortions of the pseudo-cubic unit cell, the 21 hettotypes are classified into ten diffraction types in terms of the reflection conditions for superstructure reflections (Table 4). For space 
Table 5

Diffraction groups appearing in different zone-axis CBED patterns for the hettotypes belonging to diffraction type I.

Buxton's notations are used for the diffraction groups (Buxton et al., 1976).

\begin{tabular}{|c|c|c|c|c|c|c|c|c|c|}
\hline \multirow[b]{2}{*}{$\begin{array}{l}\text { Tilt system } \\
\text { number }\end{array}$} & \multirow[b]{2}{*}{$\begin{array}{l}\text { Extended Glazer } \\
\text { notation }\end{array}$} & \multirow[b]{2}{*}{$\begin{array}{l}\text { Space } \\
\text { group }\end{array}$} & \multicolumn{7}{|c|}{ Diffraction group } \\
\hline & & & {$[001]_{\mathrm{p}}$} & {$[100]_{\mathrm{p}}$} & {$[010]_{\mathrm{p}}$} & {$[u v 0]_{\mathrm{p}}$} & {$[0 v w]_{\mathrm{p}}$} & {$[u 0 w]_{\mathrm{p}}$} & $\langle u v w\rangle_{\mathrm{p}}$ \\
\hline $1-1$ & $a^{+} b^{+} C^{+}$ & Pmmm (No. 47) & $2 m m 1_{R}$ & $2 m m 1_{R}$ & $2 m m 1_{R}$ & $2_{R} m m_{R}$ & $2_{R} m m_{R}$ & $2_{R} m m_{R}$ & $2_{R}$ \\
\hline $4-1$ & $a^{+} b^{-} C^{+}$ & $\operatorname{Pm} 2 m$ (No. 25) & $m 1_{R}$ & $m 1_{R}$ & $2 \mathrm{~mm}$ & $m$ & $m_{R}$ & $m_{R}$ & 1 \\
\hline $4-2$ & $a^{+} b^{+} C^{-}$ & Pmm2 (No. 25) & $2 \mathrm{~mm}$ & $m 1_{R}$ & $m 1_{R}$ & $m_{R}$ & $m$ & $m$ & 1 \\
\hline $5-1$ & $a^{+} a^{+} C^{-}$ & $P \overline{4} m 2$ (No. 115$)$ & $4_{R} m m_{R}$ & $2_{R} m m_{R}$ & $2_{R} m m_{R}$ & $m_{R}$ & $m_{R}$ & $m_{R}$ & 1 \\
\hline $16-1$ & $a^{+} a^{+} C^{0}$ & $\mathrm{P} 4 / \mathrm{mmm}$ (No. 123 ) & $4 m m 1_{R}$ & $2 m m 1_{R}$ & $2 m m 1_{R}$ & $2_{R} m m_{R}$ & $2_{R} m m_{R}$ & $2_{R} m m_{R}$ & $2_{R}$ \\
\hline
\end{tabular}

the presence or absence of superstructure reflections caused by octahedral tilting, the layered ordering of $A$ cations and structural absences simultaneously with reference to Table 4, in which indices of superstructure reflections observed in SAED patterns taken along some lowindexed directions are tabulated for the ten different diffraction types. Since the different diffraction types

groups that may be accompanied by structural absences, classification was made for both cases (as in I and I-A, VII and VII-A, and IX and IX-A in Table 4).

For practical electron diffraction analysis, Woodward \& Reaney (2005) and Woodward et al. (2006) have proposed the 'orientational' method, in which the occurrence of octahedral tilting along three $\langle 100\rangle_{\mathrm{p}}$ axes is examined by detecting superstructure reflections in selected-area electron diffraction (SAED) patterns taken from the same grain along $\langle 111\rangle_{\mathrm{p}}$ and three different $\langle 110\rangle_{\mathrm{p}}$ (Fig. 2). In a similar way, we can examine exhibit unique reflection conditions, they are distinguished from each other by SAED. In this circumstance, seven hettotypes belonging to the diffraction types IV-X $\left(A^{-} b^{+} c^{-}\right.$, $a^{+} b^{-} C^{0}, a^{+} b^{0} C^{-}, A^{0} b^{0} c^{+}, a^{0} a^{0} C^{+}, a^{0} a^{0} C^{-}$and $\left.a^{0} a^{0} C^{0}\right)$ can be distinguished unambiguously by conducting SAED analysis only. For hettotypes belonging to the diffraction types I-III, more information (such as axial ratios and point group) that may produce differences in positions and/or relative intensities for the superstructure reflections is needed to determine the correct space group. However, such distinctions can usually be made by taking convergent-beam electron diffraction (CBED) patterns with $\langle 100\rangle_{\mathrm{p}}$ incidences (in particular with the $[001]_{\mathrm{p}}$ incidence), since the diffraction group exhibited with a particular incidence usually differentiates hettotype. Some examples of diffraction types I-III are now described.

4.2.1. Diffraction type I. Five different hettotypes $\left(a^{+} b^{+} C^{+}\right.$, $a^{+} a^{+} C^{0}, \quad a^{+} b^{-} C^{+}, \quad a^{+} b^{+} C^{-}$and $a^{+} a^{+} C^{-}$) are classified in this diffraction type. As seen in Table 4, all possible superstructure reflections can occur for all the hettotypes, indicating the difficulty in distinguishing these space groups by SAED alone. However, since the $A$ and $B$ cations are generally more efficient electron scatterers than $X$ anions in $A B X_{3}$ perovskites, the intensity of superstructure reflections of the $1 / 2\left\{\begin{array}{llll}0 & 0 & o d d\end{array}\right\}_{\mathrm{p}}$ type caused by the layered ordering of $A$ cations, which is set to occur along the $c$ axis of the pseudo-cubic unit cell in Table 4, is expected to be higher than that of other superstructure reflections. Then, the $c$ axis of the pseudo-cubic unit cell is

Figure 2

Relationship between SAED patterns of $[111]_{\mathrm{p}},[110]_{\mathrm{p}},[101]_{\mathrm{p}}$ and $[011]_{\mathrm{p}}$ incidences. Indices with the subscript $\mathrm{p}$ refer to the pseudo-cubic unit cell corresponding to the ideal perovskite structure. 


\section{research papers}

Table 6

Diffraction groups appearing in different zone-axis CBED patterns for the hettotypes belonging to diffraction type II.

Buxton's notations are used for the diffraction groups (Buxton et al., 1976).

\begin{tabular}{|c|c|c|c|c|c|c|c|c|c|}
\hline \multirow[b]{2}{*}{$\begin{array}{l}\text { Tilt system } \\
\text { number }\end{array}$} & \multirow[b]{2}{*}{$\begin{array}{l}\text { Extended Glazer } \\
\text { notation }\end{array}$} & \multirow[b]{2}{*}{$\begin{array}{l}\text { Space } \\
\text { group }\end{array}$} & \multicolumn{7}{|c|}{ Diffraction group } \\
\hline & & & {$[001]_{\mathrm{p}}$} & {$[100]_{\mathrm{p}}$} & {$[010]_{\mathrm{p}}$} & {$[u v 0]_{\mathrm{p}}$} & {$[0 v w]_{\mathrm{p}}$} & {$[u 0 w]_{\mathrm{p}}$} & $\langle u v w\rangle_{\mathrm{p}}$ \\
\hline $8-1$ & $a^{-} b^{-} C^{+}$ & $P m($ No. $6: c)$ & $1_{R}$ & 1 & 1 & $m$ & 1 & 1 & 1 \\
\hline $12-1$ & $a^{-} b^{-} C^{-}$ & $C \overline{1}$ (No. 2) & $2_{R}$ & $2_{R}$ & $2_{R}$ & $2_{R}$ & $2_{R}$ & $2_{R}$ & $2_{R}$ \\
\hline $17-2$ & $a^{0} b^{-} C^{+}$ & Cm2m (No. 38) & $m 1_{R}$ & $m 1_{R}$ & $2 m m$ & $m$ & $m$ & $m_{R}$ & 1 \\
\hline $19-1$ & $a^{-} b^{-} C^{0}$ & $P 2 / m$ (No. 10:c) & $21_{R}$ & $2{ }_{R}$ & $2{ }_{R}$ & $2_{R} m m_{R}$ & $2{ }_{R}$ & $2_{R}$ & $2_{R}$ \\
\hline $19-2$ & $a^{0} b^{-} C^{-}$ & $C 2 / m($ No. $12: a 2)$ & $2_{R}$ & $21_{R}$ & $2{ }_{R}$ & $2{ }_{R}$ & $2_{R} m m_{R}$ & $2_{R}$ & $2_{R}$ \\
\hline $22-1$ & $a^{0} b^{-} C^{0}$ & Cmmm (No. 65) & $2 m m 1_{R}$ & $2 m m 1_{R}$ & $2 m m 1_{R}$ & $2_{R} m m_{R}$ & $2_{R} m m_{R}$ & $2_{R} m m_{R}$ & $2_{R}$ \\
\hline
\end{tabular}

\section{Table 7}

Diffraction groups appearing in different zone-axis CBED patterns for the hettotypes belonging to diffraction type III.

Buxton's notations are used for the diffraction groups (Buxton et al., 1976).

\begin{tabular}{lllllllllll}
\hline & \multicolumn{10}{c}{ Diffraction group } \\
\cline { 3 - 9 } $\begin{array}{l}\text { Tilt system } \\
\text { number }\end{array}$ & $\begin{array}{l}\text { Extended Glazer } \\
\text { notation }\end{array}$ & $\begin{array}{l}\text { Space } \\
\text { group }\end{array}$ & {$[001]_{\mathrm{p}}$} & {$[100]_{\mathrm{p}}$} & {$[010]_{\mathrm{p}}$} & {$[u v 0]_{\mathrm{p}}$} & {$[0 v w]_{\mathrm{p}}$} & {$[u 0 w]_{\mathrm{p}}$} & $\langle u v w\rangle_{\mathrm{p}}$ \\
\hline $10-1$ & $a^{-} a^{-} C^{+}$ & $P b 2_{1} m$ (No. 26) & $m 1_{R}$ & $m 1_{R}$ & $2 m m$ & $m$ & $m$ & $m_{R}$ & 1 \\
$13-1$ & $a^{-} a^{-} C^{-}$ & $P 2 / \mathrm{c}$ (No. 13:b1) & $2_{R}$ & $2_{R}$ & $21_{R}$ & $2_{R}$ & $2_{R}$ & $2_{R} m m_{R}$ & $2_{R}$ \\
$20-1$ & $a^{-} a^{-} C^{0}$ & $P b m m$ (No. 51) & $2 m m 1_{R}$ & $2 m m 1_{R}$ & $2 m m 1_{R}$ & $2_{R} m m_{R}$ & $2_{R} m m_{R}$ & $2_{R} m m_{R}$ & $2_{R}$ \\
\hline
\end{tabular}
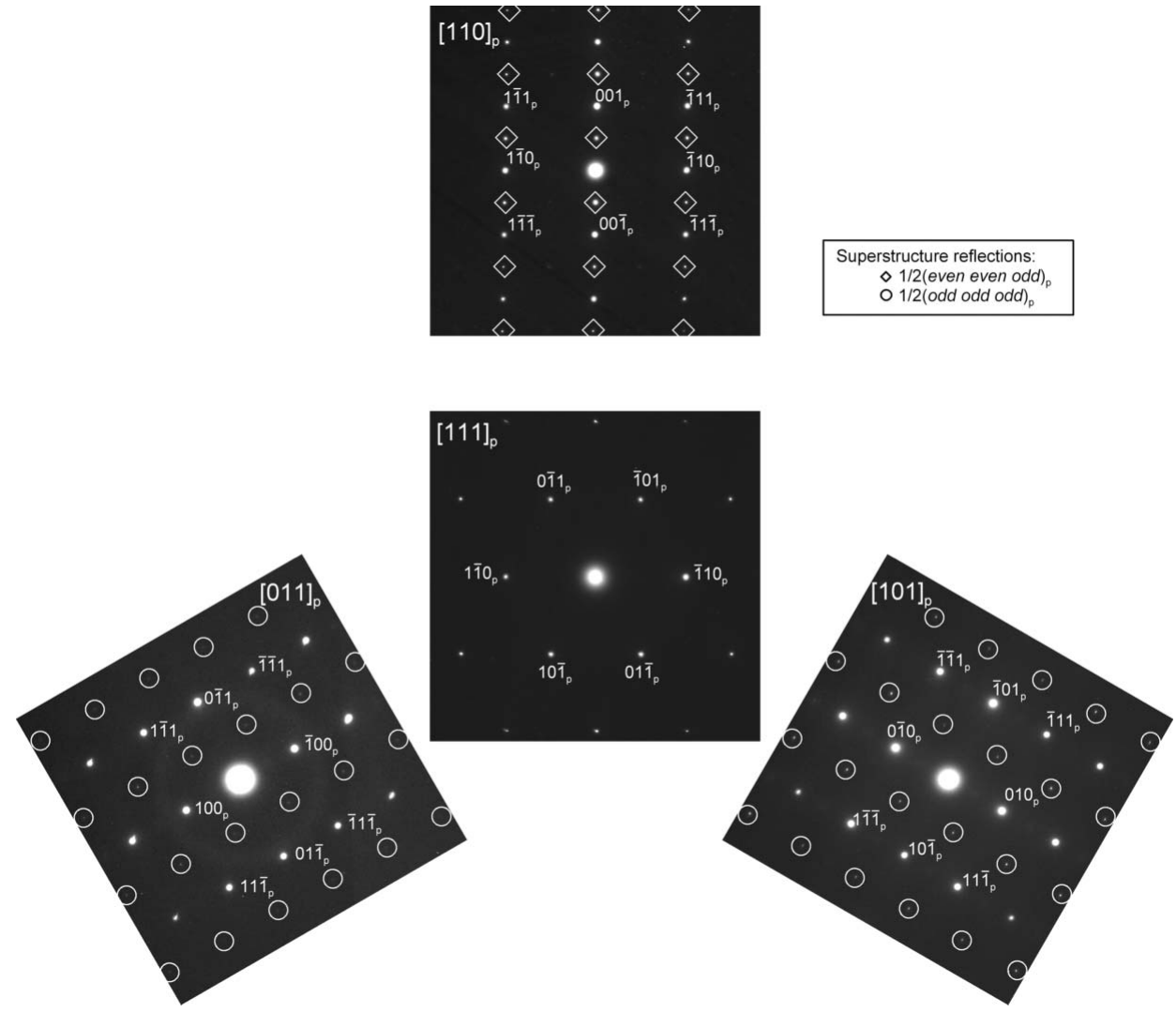

Figure 3

SAED patterns taken from Li-rich LLT with a chemical composition of $\mathrm{La}_{0.57} \mathrm{Li}_{0.3} \mathrm{TiO}_{3}$ projected along $[111]_{p},[110]_{p},[101]_{p}$ and $[011]_{p}$ incidences. Indices with the subscript $p$ refer to the pseudo-cubic unit cell corresponding to the ideal perovskite structure. unambiguously designated from the observation of the most intense superstructure reflection of the 1/2(001) type in SAED patterns. In practice, the relative intensity of the corresponding superstructure reflections [with indices of $1 / 2(100)_{\mathrm{p}}, 1 / 2(010)_{\mathrm{p}}$ and $1 / 2(001)_{\mathrm{p}}$ ] appearing in SAED patterns is compared with that of three $\langle 110\rangle_{\mathrm{p}}$ directions. Once the $c$ axis is unambiguously designated, CBED patterns, in particular those with $[001]_{\mathrm{p}}$ incident, are examined to distinguish space groups. In the case of diffraction type $I$, the diffraction groups appearing in CBED patterns of all the hettotypes with the $[001]_{\mathrm{p}}$ incident differ from each other (Table 5). Then, the appropriate space group is determined by inspecting the symmetry of a CBED pattern taken along the [001] zone-axis (Buxton et al., 1976; Tanaka, Saito \& Sekii, 1983; Tanaka, Sekii \& Nagasawa, 1983; Tanaka et al., 1988; Tanaka \& Terauchi, 1985; Spence \& Zuo, 1992).

4.2.2. Diffraction type II. For all the hettotypes $\left(a^{-} b^{-} C^{+}, a^{-} b^{-} C^{-}\right.$, $a^{0} b^{-} C^{+}, \quad a^{0} b^{-} C^{-}, \quad a^{0} b^{-} C^{0}$ and $a^{-} b^{-} C^{0}$ ) belonging to this diffraction type, the $c$ axis of the pseudocubic unit cell is readily identified from the superstructure reflection $1 / 2(001)_{\mathrm{p}}$ type in the $[110]_{\mathrm{p}}$ SAED pattern, since superstructure reflections of the $1 / 2(010)_{\mathrm{p}}$ and $1 /$ $2(100)_{\mathrm{p}}$ types do not appear in SAED patterns with $[101]_{\mathrm{p}}$ or $[011]_{\mathrm{p}}$ incident, respectively (Table 4). When judged from Table 6 , in which diffraction groups appearing in CBED patterns with the incidence along some lowindex directions are listed for the six hettotypes, the symmetry analysis along the [001] direction is found to be the most effective in space-group determination, except for $C \overline{1}$ and $C 2 / m$. To differentiate these symmetries, additional CBED analysis along $[100]_{\mathrm{p}}$ or $[0 v w]_{p}$ is needed.

4.2.3. Diffraction type III. As in the case of diffraction type II, 
Table 8

CBED pattern symmetries for diffraction groups listed in Tables 5, 6 and 7.

Where a dash appears, the special symmetries can be deduced from columns 5 and 6 of this table (Buxton et al., 1976).

\begin{tabular}{|c|c|c|c|c|c|c|c|}
\hline \multirow[b]{2}{*}{$\begin{array}{l}\text { Diffraction } \\
\text { group }\end{array}$} & \multirow{2}{*}{$\begin{array}{l}\text { Bright } \\
\text { field } \\
(\mathrm{BP})\end{array}$} & \multirow{2}{*}{$\begin{array}{l}\text { Whole } \\
\text { pattern } \\
(\mathrm{WP})\end{array}$} & \multicolumn{2}{|c|}{ Dark field (DP) } & \multicolumn{2}{|l|}{$\pm \mathrm{DP}$} & \multirow{2}{*}{$\begin{array}{l}\text { Projection } \\
\text { diffraction } \\
\text { group }\end{array}$} \\
\hline & & & General & Special & General & Special & \\
\hline 1 & 1 & 1 & 1 & None & 1 & None & $1_{R}$ \\
\hline $1_{R}$ & 2 & 1 & 2 & None & 1 & None & $1_{R}$ \\
\hline $2_{R}$ & 1 & 1 & 1 & None & $2_{R}$ & None & $21_{R}$ \\
\hline $21_{R}$ & 2 & 2 & 2 & None & $21_{R}$ & None & $21_{R}$ \\
\hline$m_{R}$ & $m$ & 1 & 1 & $m$ & 1 & $m_{R}$ & $m 1_{R}$ \\
\hline$m$ & $m$ & $m$ & 1 & $m$ & 1 & $m$ & $m 1_{R}$ \\
\hline$m 1_{R}$ & $2 \mathrm{~mm}$ & $m$ & 2 & $m$ & 1 & $m 1_{R}$ & $m 1_{R}$ \\
\hline $2 \mathrm{~mm}$ & $2 \mathrm{~mm}$ & $2 \mathrm{~mm}$ & 1 & $m$ & 2 & - & $2 m m 1_{R}$ \\
\hline $2_{R} m m_{R}$ & $m$ & $m$ & 1 & $m$ & $2_{R}$ & - & $2 m m 1_{R}$ \\
\hline $2 m m 1_{R}$ & $2 \mathrm{~mm}$ & $2 \mathrm{~mm}$ & 2 & $2 \mathrm{~mm}$ & $21_{R}$ & - & $2 m m 1_{R}$ \\
\hline $4_{R} m m_{R}$ & $4 m m$ & $2 \mathrm{~mm}$ & 1 & $m$ & 2 & - & $4 m m 1_{R}$ \\
\hline $4 m m 1_{R}$ & $4 m m$ & $4 m m$ & 2 & $2 \mathrm{~mm}$ & $21_{R}$ & - & $4 m m 1_{R}$ \\
\hline
\end{tabular}

superstructure reflections of the $1 / 2\{00 \text { odd }\}_{\mathrm{p}}$ type caused by the layered ordering of $A$ cations along the $c$ axis appear only in $[110]_{\mathrm{p}}$ SAED patterns for all the hettotypes $\left(a^{-} a^{-} C^{+}\right.$, $a^{-} a^{-} C^{-}$and $a^{-} a^{-} C^{0}$ ) and, hence, the $c$ axis of the pseudocubic unit cell is unambiguously designated from the obser-

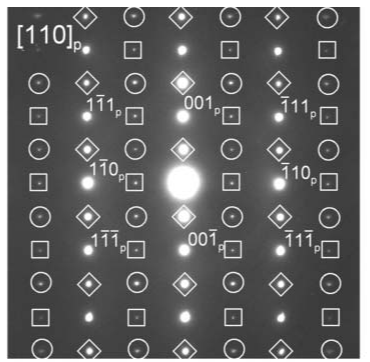

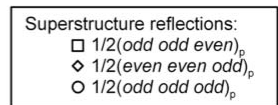

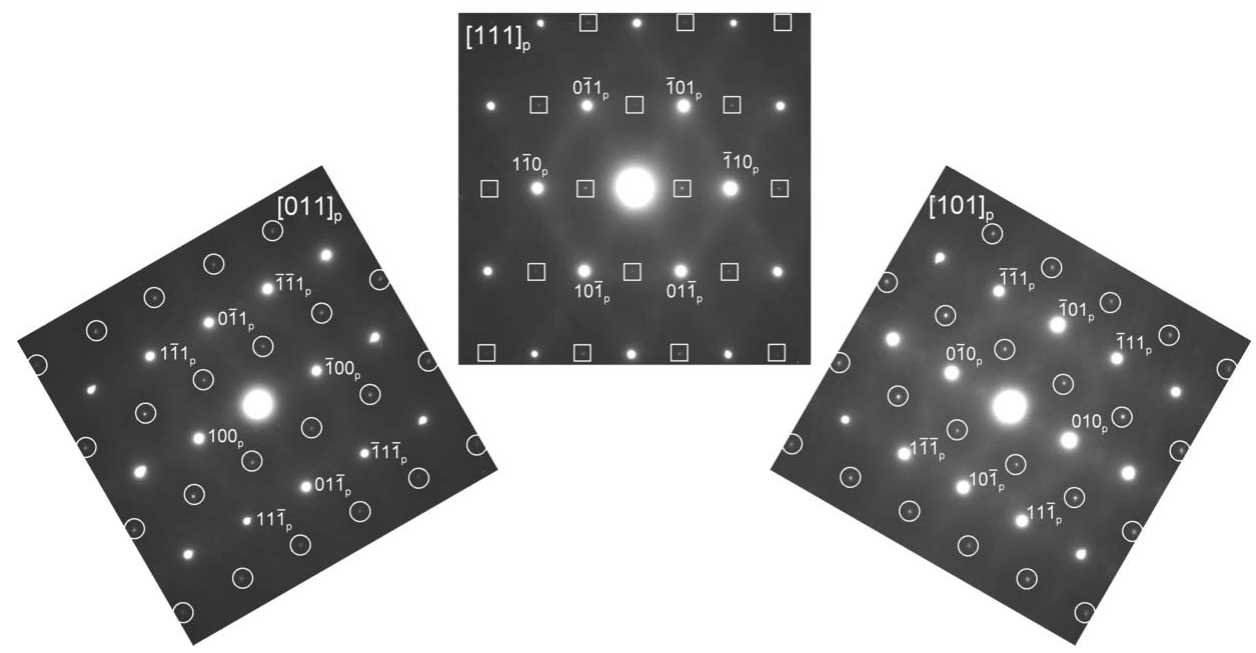

Figure 4

SAED patterns taken from Li-poor LLT with a chemical composition of $\mathrm{La}_{0.64} \mathrm{Li}_{0.09} \mathrm{TiO}_{3}$ projected along $[111]_{\mathrm{p}},[110]_{\mathrm{p}},[101]_{\mathrm{p}}$ and $[011]_{\mathrm{p}}$ incidences. Indices with the subscript $\mathrm{p}$ refer to the pseudo-cubic unit cell corresponding to the ideal perovskite structure. vation of the $1 / 2(001)_{\mathrm{p}}$-type superstructure reflection. Distinction of space groups can be readily made by the symmetry analysis of $\langle 001\rangle_{p}$ CBED patterns (Table 7).

\section{Applications of the present method to real perovskites}

The crystal structure of lithium lanthanum titanate, $\mathrm{La}_{2 / 3-x} \mathrm{Li}_{3 x} \mathrm{TiO}_{3}$ (LLT) varies with chemical composition, synthesis method and heat treatment (Stramare et al., 2003). Since the contribution of $\mathrm{Li}$ atoms to electron diffraction intensities is extremely small, LLT can be regarded to a first approximation as a simple $A$-site deficient perovskite. The results of crystal structure determination for LLT with two different compositions, $\mathrm{La}_{0.57} \mathrm{Li}_{0.3} \mathrm{TiO}_{3}(x=0.1$ : $\mathrm{Li}$ rich $)$ and $\mathrm{La}_{0.64} \mathrm{Li}_{0.09} \mathrm{TiO}_{3}(x=0.03$ : Li poor $)$ are presented below. The Li-rich sample was grown by the Tammann-Stöber method, then heat treated at $1073 \mathrm{~K}$ for $24 \mathrm{~h}$. The Li-poor sample was prepared by conventional solid-state reaction. The details of sample preparation are described by Kishida et al. $(2007 a, b)$.

\section{1. $\mathrm{La}_{0.57} \mathrm{Li}_{0.3} \mathrm{TiO}_{3}(x=0.1$; Li rich $)$}

SAED patterns were taken from the same very thin area along $[111]_{p}$ and $\langle 110\rangle_{p}$ (Fig. 3). Superstructure reflections of the $1 / 2\{001\}_{p}$ type are clearly observed in the $[110]_{p}$ SAED pattern but not in other orientations, confirming the existence of a layered $A$-cation ordering along $[001]_{\mathrm{p}}$. No superstructure reflections are observed in the $[111]_{\mathrm{p}}$ pattern. Superstructure reflections of the $1 / 2$ (even even odd $)_{\mathrm{p}}$ type are observed in $[110]_{\mathrm{p}}$, while those of the $1 / 2(\text { odd } \text { odd } \text { odd })_{\mathrm{p}}\left(h_{\mathrm{p}} \neq \pm k_{\mathrm{p}}\right)$ type are observed in $[011]_{\mathrm{p}}$ and $[101]_{\mathrm{p}}$. Among the ten diffraction types in Table 4, the reflection conditions for the hettotype of $a^{0} a^{0} C^{-}$accompanied by the special arrangements of the $X$ anions, which give rise to structural absences due to the body-centring as described in $\$ 4.1$, perfectly coincide with these observations. The space group P4/nbm (No. 125, origin choice 2) is thus determined for Li-rich LLT purely by SAED analysis. This conclusion is consistent with our previous result (Kishida et al., 2007a) and with that of neutron diffraction (Catti et al., 2007). The reflection conditions clearly support the occurrence of structural absences due to the body-centring of $X$ anions in $8 m$ sites: $(x+1 / 2, x, z)$ taking a special 


\section{research papers}

value $(1 / 4)$ of the $z$ coordinate as described in $\S 4.1$. This indicates that the aristotype $(14 / \mathrm{mcm})$ remains body-centred even after the introduction of $A$-cation ordering and that great care must be taken in assigning atomic coordinates when the occurrence of structural absences is highly probable. It is important to note that body-centring would also be generated with the $B$ cations in $4 g$ sites by taking a special value (1/4) of the $z$ coordinate $[4 g$ sites: $(3 / 4,3 / 4, z)]$ in a tilt system $a^{0} a^{0} C^{-}$ with undistorted $B X_{6}$ octahedra. However, it is difficult to confirm the occurrence of the body-centring for the $B$ cations simply by considering the reflection conditions, since the $B$ cations in $4 g$ sites already possess the $C$-centring giving rise to the special condition of $h k l: h+k=2 n$ that yields additional reflection conditions.
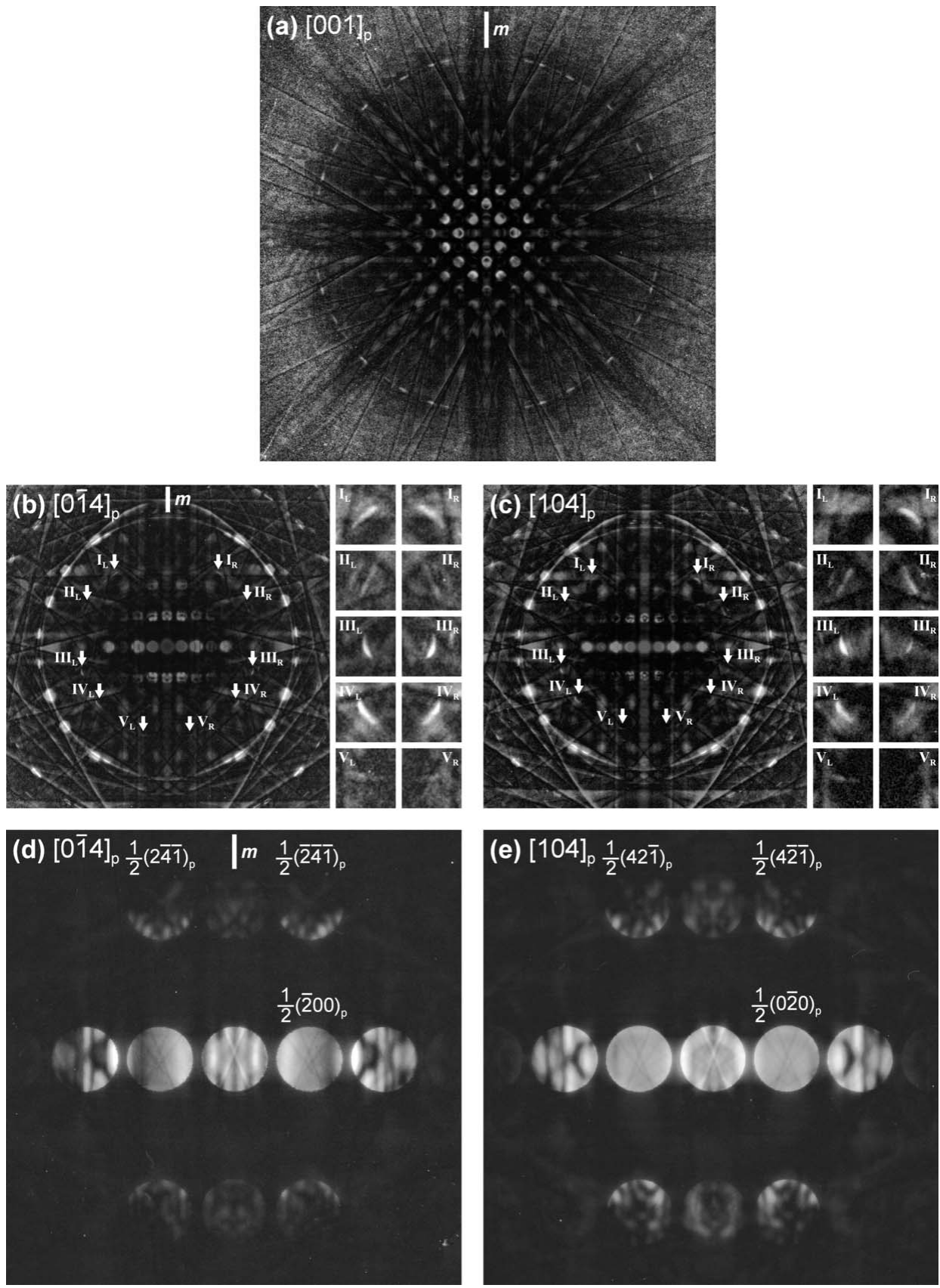

\section{Figure 5}

(a) $[001]_{\mathrm{p}},(b),(d)[014]_{\mathrm{p}}$ and $(c),(e)[104]_{\mathrm{p}}$ CBED patterns of Li-poor LLT with a chemical composition of $\mathrm{La}_{0.64} \mathrm{Li}_{0.09} \mathrm{TiO}_{3}$. The background is subtracted from the original image to enhance the contrast from the higher-order Laue zone (HOLZ) reflections for $(a),(b)$ and $(c)$. Some of the first-order Laue zone (FOLZ) reflections marked as $\mathrm{I}_{\mathrm{L}} \sim \mathrm{V}_{\mathrm{L}}$ and $\mathrm{I}_{\mathrm{R}} \sim \mathrm{V}_{\mathrm{R}}$ in $(b)$ and $(c)$ are enlarged and indicated as insets. Indices with the subscript $\mathrm{p}$ refer to the pseudo-cubic unit cell corresponding to the ideal perovskite structure.

\section{2. $\mathrm{La}_{0.64} \mathrm{Li}_{0.09} \mathrm{TiO}_{3}(x=0.03 ; \mathrm{Li}$ poor)}

SAED patterns from a very thin area along $[111]_{\mathrm{p}}$ and $\langle 110\rangle_{\mathrm{p}}$ are shown in Fig. 4. Superstructure reflections observed in $[111]_{\mathrm{p}}$ are assigned to the $1 / 2\{$ odd odd even $\}_{\mathrm{p}}$ type, assuming that the layered ordering of $A$ cations occurs along the $c$ axis of the ideal perovskite structure. Three types of superstructure reflections observed in $[110]_{\mathrm{p}}$ are indexed as those of the $1 / 2(\text { odd odd odd })_{\mathrm{p}}, 1 /$ 2 (odd odd even $)_{\mathrm{p}}$ and $1 / 2$ (even even odd $)_{\mathrm{p}}$ types, while those reflections in $[011]_{\mathrm{p}}$ and $[101]_{\mathrm{p}}$ are indexed as the $1 / 2$ (odd odd odd $)_{\mathrm{p}}$ type. These conditions indicate that the space group belongs to diffraction type II (Table 4). Distinction of the seven space groups in the diffraction type II can be made by CBED analysis. A CBED pattern taken along $[001]_{\mathrm{p}}$ is shown in Fig. 5(a). In $[001]_{\mathrm{p}}$, five different diffraction groups, namely $1_{R}, 2_{R}, 21_{R}, m 1_{R}$ and $2 m m 1_{R}$ are possible for the seven space groups in the diffraction type II (Table 6). The five diffraction groups can be distinguished theoretically by inspecting the symmetry information appearing in the brightfield pattern (BP) and whole pattern (WP) in the [001 $]_{\mathrm{p}}$ CBED pattern (Table 8). However, the intensity details of the first-order Laue zone (FOLZ) reflections in the WP were too weak to obtain such detailed information. Therefore, CBED was performed along the $[0 \overline{1} 4]_{\mathrm{p}}$ and $[104]_{\mathrm{p}}$ zoneaxis orientations, which are close to the $[001]_{\mathrm{p}}$ zone (Figs. $5 b$ and $c$ ) to locate a mirror plane on $(100)_{\mathrm{p}}$ and $(010)_{\mathrm{p}}$. The WP of the $[0 \overline{1} 4]_{\mathrm{p}}$ 
zone indicates the existence of a mirror plane on $(100)_{\mathrm{p}}$, while the mirror was not with [104] $]_{\mathrm{p}}$ incident. Thus, in $[014]_{\mathrm{p}} \mathrm{Cm} 2 \mathrm{~m}$, $\mathrm{C2} / \mathrm{m}$ and $\mathrm{Cmmm}$ are possible space groups, while [104 $]_{\mathrm{p}}$ clearly demonstrates the space group is $C m 2 m$ or $C 2 / m$. The distinction between $C m 2 m$ and $C 2 / m$ can be easily made by inspecting whether a mirror appears in the BP (Table 8). Since the mirror symmetry is clearly observed in $[104]_{\mathrm{p}} \mathrm{BP}$ taken from relatively thick regions (Fig. 5e), the space group for this LLT phase is determined to be $C m 2 m$. This result differs from that previously reported for $A$-site deficient perovskites with similar compositions (Carrillo et al., 2000; Inaguma et al., 2002; Yashima et al., 2005). The reason for the discrepancy is believed to be due to the fact that these workers discarded $\mathrm{Cm} 2 \mathrm{~m}$ (standard notation: Amm2) simply because it has a lower symmetry than $\mathrm{Cmmm}$.

\section{Conclusions}

Space groups of $A B X_{3}$ perovskites with both tilted $B X_{6}$ octahedra and layered ordering of $A$ cations are examined on the basis of group-subgroup relations. The possibility of distinguishing the space groups by electron diffraction is also examined. The results are summarized as follows:

(i) By introducing layered ordering of $A$ cations to the previously deduced 16 different types of $A B X_{3}$ perovskites accompanied by the tilting of $B X_{6}$ octahedra, 21 different types of perovskite structures are generated. Analysis based on group-subgroup relations allows assignment of 16 different space groups to the 21 perovskite structures.

(ii) These 21 perovskites (with 16 different space groups) are classified into ten diffraction types with respect to the conditions for superstructure reflections caused by the layered ordering of $A$ cations, the tilting of $B X_{6}$ octahedra and structural absences.

(iii) Of the 21 perovskite structures, seven structures (belonging to seven diffraction types) can be distinguished by the analysis of SAED patterns. For the other 14 structures (belonging to three diffraction types), additional analysis by CBED is necessary for space-group determination. The space groups of lithium lanthanum titanate with two different perovskite structures (chemical compositions) were successfully deduced by electron diffraction experiments.

This work was supported by a Grant-in-Aid for Young Scientists (A) (grant No. 21686066) and a Grant-in-Aid for Exploratory Research (grant No. 19656179) from the Ministry of Education, Culture, Sports, Science and Technology (MEXT), Japan, and in part by the Global COE (Center of Excellence) Program of the International Center for Integrated Research and Advanced Education in Materials Science from the MEXT, Japan.

\section{References}

Aroyo, M. I., Perez-Mato, J. M., Capillas, C., Kroumova, E., Ivantchev, S., Madariaga, G., Kirov, A. \& Wondratschek, H. (2006). Z. Kristallogr. 221, 15-27.

Buxton, B. F., Eades, J. A., Steeds, J. W. \& Rackham, G. M. (1976). Philos. Trans. R. Soc. London Ser. A, 281, 171-194.

Carrillo, L., Villafuerte-Castrejon, M. E., Gonzalez, G., Sansores, L. E., Bucio, L., Duque, J. \& Pomes, R. (2000). J. Mater. Sci. 35, 3047-3052.

Catti, M., Sommariva, M. \& Ibberson, R. M. (2007). J. Mater. Chem. 17, 1300-1307.

Cava, R. J. (1990). Science, 247, 656-662.

Davies, P. K. (1999). Curr. Opin. Solid State Mater. Sci. 4, 467-471.

Glazer, A. M. (1972). Acta Cryst. B28, 3384-3392.

Glazer, A. M. (1975). Acta Cryst. A31, 756-762.

Goodenough, J. B. (2004). Rep. Prog. Phys. 67, 1915-1993.

Hahn, T. (2002). Editor. International Tables for Crystallography, Vol. A, Space-Group Symmetry, 5th revised ed. Dordecht: Kluwer Academic Publishers.

Howard, C. J., Kennedy, B. J. \& Woodward, P. M. (2003). Acta Cryst. B59, 463-471.

Howard, C. J. \& Stokes, H. T. (1998). Acta Cryst. B54, 782-789.

Howard, C. J. \& Stokes, H. T. (2004). Acta Cryst. B60, 674-684.

Howard, C. J. \& Zhang, Z. (2004). Acta Cryst. B60, 249-251.

Inaguma, Y., Chen, L., Itoh, M., Nakamura, T., Uchida, T., Ikuta, H. \& Wakihara, M. (1993). Solid State Commun. 86, 689-693.

Inaguma, Y., Katsumata, T., Itoh, M. \& Morii, Y. (2002). J. Solid State Chem. 166, 67-72.

Kishida, K., Miyata, M., Wada, N., Okamoto, N. L., Tanaka, K., Inui, H., Koyama, H., Hattori, T., Iriyama, Y. \& Ogumi, Z. (2007a). J. Electron Microsc. 56, 225-234.

Kishida, K., Miyata, M., Wada, N., Okamoto, N. L., Tanaka, K., Inui, H., Koyama, H., Hattori, T., Iriyama, Y. \& Ogumi, Z. (2007b). Acta Mater. 55, 4713-4722.

Leinenweber, K. \& Parise, J. (1995). J. Solid State Chem. 114, 277-281.

Megaw, H. D. (1973). Crystal Structures - A Working Approach. Philadelphia: W. B. Saunders.

Mitchell, R. H. (2002). Perovskites: Modern and Ancient. Ontario: Almaz Press Inc.

Nakayama, M., Ikuta, H., Uchimoto, Y., Wakihara, M., Terada, Y., Miyanaga, T. \& Watanabe, I. (2003). J. Phys. Chem. B, 107, 1071510721.

Newnham, R. E. \& Ruschau, G. R. (1991). J. Am. Ceram. Soc. 74, 463-480.

Spence, J. C. H. \& Zuo, J. M. (1992). Electron Microdiffraction. New York: Plenum Press.

Stramare, S., Thangadurai, V. \& Weppner, W. (2003). Chem. Mater. 15, 3974-3990.

Tanaka, M., Saito, R. \& Sekii, H. (1983). Acta Cryst. A39, 357-368.

Tanaka, M., Sekii, H. \& Nagasawa, T. (1983). Acta Cryst. A39, 825837.

Tanaka, M. \& Terauchi, M. (1985). Convergent-Beam Electron Diffraction. Tokyo: Jeol Ltd.

Tanaka, M., Terauchi, M. \& Kaneyama, T. (1988). Convergent-Beam Electron Diffraction II. Tokyo: Jeol Ltd.

Woodward, D. I. \& Reaney, I. M. (2005). Acta Cryst. B61, 387-399.

Woodward, D. I., Wise, P. L., Lee, W. E. \& Reaney, I. M. (2006). J. Phys. Condens. Matter, 18, 2401-2408.

Woodward, P. M. (1997a). Acta Cryst. B53, 32-43.

Woodward, P. M. (1997b). Acta Cryst. B53, 44-66.

Yashima, M., Itoh, M., Inaguma, Y. \& Morii, Y. (2005). J. Am. Ceram. Soc. 127, 3491-3495. 